\title{
Discrete Inequalities on LCT
}

\author{
Guanlei Xu1, Xiaotong Wang ${ }^{2}$, Xiaogang $\mathrm{Xu}^{2}$ \\ ${ }^{1}$ Ocean Department of Dalian Naval Academy, Dalian, China \\ ${ }^{2}$ Navgation Department of Dalian Naval Academy, Dalian, China \\ Email: xgl 86@163.com
}

Received 12 April 2015; accepted 12 May 2015; published May 2015

Copyright (C) 2015 by authors and Scientific Research Publishing Inc.

This work is licensed under the Creative Commons Attribution International License (CC BY).

http://creativecommons.org/licenses/by/4.0/

(c) (i) Open Access

\begin{abstract}
Linear canonical transform (LCT) is widely used in physical optics, mathematics and information processing. This paper investigates the generalized uncertainty principles, which plays an important role in physics, of LCT for concentrated data in limited supports. The discrete generalized uncertainty relation, whose bounds are related to LCT parameters and data lengths, is derived in theory. The uncertainty principle discloses that the data in LCT domains may have much higher concentration than that in traditional domains.
\end{abstract}

\section{Keywords}

\section{Linear Canonical Transform (LCT), Uncertainty Inequality}

\section{Introduction}

In physics, the uncertainty principle plays an important role in elementary fields, and data concentration is often considered carefully via the uncertainty principle [1]-[8]. In continuous signals, the supports are assumed to be $(-\infty,+\infty)$, based on which various uncertainty relations [1] [2] [9]-[21] have been presented. However, in practice, both the supports of time and frequency are often limited. In such case, the support $(-\infty,+\infty)$ fails to hold true. In limited supports, some papers such as [22]-[25] have discussed the uncertainty principle in conventional time-frequency domains for continuous and discrete cases and some conclusions are achieved. However, none of them has covered the linear canonical transform (LCT) in terms of Heisenberg uncertainty principles that have been widely used in various fields [4]-[6]. Therefore, there has a great need to discuss the uncertainty relations in LCT domains. As the generalization of the traditional FT, FRFT [5] [6] [26]-[28] and so on, LCT has some special properties with more transform parameters (or freedoms) and sometimes yields the better result [29]. Readers can see more details on LCT in [6] and so on. 


\section{Preliminaries}

\subsection{Definition of LCT}

Before discussing the uncertainty principle, we will introduce some relevant preliminaries. Here, we first briefly review the definition of LCT. For given continuous signal $x(t) \in L^{1}(R) \cap L^{2}(R)$ and $\|x(t)\|_{2}=1$, its LCT [6] is defined as

$$
\begin{aligned}
X_{(a, b, c, d)}(u) & =F^{(a, b, c, d)}(x(t))=\int_{-\infty}^{\infty} x(t) K_{(a, b, c, d)}(u, t) \mathrm{d} t \\
& = \begin{cases}\sqrt{\frac{1}{2 b \pi}} \cdot \mathrm{e}^{\frac{i d u^{2}}{2 b}} \int_{-\infty}^{\infty} \mathrm{e}^{\frac{-i u t}{b}} \mathrm{e}^{\frac{i a t^{2}}{2 b}} x(t) \mathrm{d} t, & b \neq 0, a d-b c=1 ; \\
\sqrt{d} \cdot \mathrm{e}^{\frac{i c d u^{2}}{2}} x(d u), & b=0 .\end{cases}
\end{aligned}
$$

where $n \in \mathrm{Z}$ and $i$ is the complex unit, $(a, b, c, d)$ are the transform parameters defined as that in [6]. In addition, $F^{(a 2, b 2, c 2, d 2)} F^{(a 1, b 1, c 1, d 1)}(x(t))=F^{(a, b, c, d)}(x(t))$ and $\left[\begin{array}{ll}a & b \\ c & d\end{array}\right]=\left[\begin{array}{ll}a_{2} & b_{2} \\ c_{2} & d_{2}\end{array}\right]\left[\begin{array}{ll}a_{1} & b_{1} \\ c_{1} & d_{1}\end{array}\right]$. If $\left[\begin{array}{ll}1 & 0 \\ 0 & 1\end{array}\right]=\left[\begin{array}{ll}a_{2} & b_{2} \\ c_{2} & d_{2}\end{array}\right]\left[\begin{array}{ll}a_{1} & b_{1} \\ c_{1} & d_{1}\end{array}\right]$, then $F^{(a 1, b 1, c 1, d 1)}(\bullet)$ and $F^{(a 2, b 2, c 2, d 2)}(\bullet)$ are the LCT transform pairs, i.e., $F^{(a 2, b 2, c 2, d 2)} F^{(a 1, b 1, c 1, d 1)}(x(t))=x(t)$. Also, if $(a, b, c, d)=(0,1,-1,0)$, we have the following equations:

$$
\begin{aligned}
F^{(0,1,-1,0)}(x(t)) & =X(u) \\
& =\int_{-\infty}^{\infty} x(t) K_{(0,1,-1,0)}(u, t) \mathrm{d} t \text { and } x(t)=\sqrt{\frac{1}{2 \pi}} \cdot \int_{-\infty}^{\infty} \mathrm{e}^{\frac{i u t}{b}} X(u) \mathrm{d} t . \\
& =\sqrt{\frac{1}{2 \pi}} \cdot \int_{-\infty}^{\infty} \mathrm{e}^{\frac{-i u t}{b}} x(t) \mathrm{d} t,
\end{aligned}
$$

However, unlike the discrete FT, there are a few definitions for the DLCT (discrete LCT), but not only one. In this paper, we will employ the definition defined as follows [6]:

$$
\begin{aligned}
\hat{x}_{(a, b, c, d)}(k) & =\sum_{n=1}^{N} \sqrt{1 / i N b} \cdot \mathrm{e}^{\frac{i d k^{2}}{2 b}} \mathrm{e}^{\frac{-i k n}{N b}} \mathrm{e}^{\frac{i n^{2} a}{2 b N^{2}}} \tilde{x}(n) \\
& =\sum_{n=1}^{N} u_{(a, b, c, d)}(k, n) \cdot \tilde{x}(n), \quad 1 \leq n, k \leq N .
\end{aligned}
$$

Clearly, if $(a, b, c, d)=(1,0,0,1)$, (2) reduces to the traditional discrete FT [6]. Also, we can rewrite definition (2) as $\hat{X}_{A}=U_{A} \tilde{X}$ with $A=(a, b, c, d)$ and $A^{-1} A=I$, where $U_{A}=\left[u_{A}(k, n)\right]_{N \times N}, \tilde{X}=[\tilde{x}(n)]_{N \times 1}$.

For DLCT, we have the following property [5] [6]:

$$
\left\|\hat{X}_{A}\right\|_{2}=\left\|U_{A} \tilde{X}\right\|_{2}=1 .
$$

More details on DLCT can be found in [6].

\subsection{Frequency-Limiting Operators}

Definition 1: Let $x(t)$ be a complex-valued signal with $\|x(t)\|_{L^{2}(R)}=1$ and its LCT $X_{A}(u)$, if there is a function $G_{A}(u)$ vanishing outside $W_{A}\left(W_{A}\right.$ is a measurable set) such that $\left\|X_{A}(u)-G_{A}(u)\right\|_{L^{2}(R)} \leq \varepsilon_{A}$, then $X_{A}(u)$ is $\varepsilon_{A}$-concentrated.

Specially, if $A=(1,0,0,1)$, then definition 1 reduces to the case in time domain [22] [23]. If $A=(0,1,-1,0)$, then definition 1 reduces to the case in traditional frequency domain [22] [23]. 
Definition 2: Generalized frequency-limiting operator $P_{W_{A}}$ is defined as

$$
\left(P_{W_{A}} x\right)(t) \equiv \int_{W_{A}} X_{A}(u) K_{A^{-1}}(u, t) \mathrm{d} u, X_{A}(u)=F_{A}(x(t)) .
$$

If $A=(1,0,0,1)$, then definition 2 is the time-limiting operator [22] [23]. If $A=(0,1,-1,0)$, then definition 2 is the traditional frequency-limiting operator [22] [23]. Definitions 1 and 2 disclose the relation between $\varepsilon_{A}$ and $W_{A}$. For the discrete case, we have the following definitions.

Definition 3: Let $\tilde{x}(n) \in l^{2}(R) \quad(n=1, \cdots, N)$ be a discrete sequence with $\|\tilde{x}(n)\|_{l^{2}(R)}=1$ and its DLCT $\hat{x}_{A}(k)$, if there is a sequence $\hat{x}_{A}^{\prime}(k)$ satisfying $\left\|\hat{x}_{\alpha}^{\prime}(k)\right\|_{0}=N_{A}$ such that $\left\|\hat{x}_{A}(k)-\hat{x}_{A}^{\prime}(k)\right\|_{l^{2}(R)} \leq \varepsilon_{A}$, then $\hat{x}(k)$ is $\varepsilon_{A}$-concentrated. Here, \|\|$_{0}$ is the 0 -norm operator that counts the non-zero elements.

Definition 4: Generalized discrete frequency-limiting operator $P_{N_{A}}$ is defined as $\left(P_{N_{A}} \tilde{x}\right)(n)=\sum_{k=1}^{N} \chi_{N_{A}} \hat{x}(k) u_{A^{-1}}(k, n)$ with $\hat{x}(k)$ is the DLCT of $\tilde{x}(n)$ and $\chi_{N_{A}}$ is the character function on $N_{A}\left(N_{A} \leq N\right)$.

Clearly, definitions 3 and 4 are the discrete extensions of definitions 1 and 2 . They have the similar physical meaning. These definitions are introduced for the first time, the traditional cases [22] [23] are only their special cases. Definition 3 and 4 disclose the relation between $\varepsilon_{A}$ and $N_{A}$.

\section{The Uncertainty Relations}

\subsection{The Uncertainty Principle}

First let us introduce a lemma.

$$
\text { Lemma 3: }\left\|P_{N_{A}} P_{N_{B}}\right\|_{F}=\sqrt{\frac{N_{A} \cdot N_{B}}{N\left|a_{1} b_{2}-a_{2} b_{1}\right|}}
$$

where $\|\cdot\|_{F}$ is the Frobenius matrix norm.

Proof: From the definition of the operator $P_{N_{A}} P_{N_{B}}$ in definition 4, we have

$$
\left(P_{N_{A}} P_{N_{B}} \tilde{x}\right)(n)=\sum_{k=1}^{N} \chi_{N_{A}} u_{A}(k, n) \sum_{v=1}^{N} \chi_{N_{B}} \hat{x}(v) u_{B^{-1}}(k, v) .
$$

Exchange the locations of the sum operators, we obtain

$$
\begin{aligned}
\left(P_{N_{A}} P_{N_{B}} \tilde{x}\right)(n) & =\sum_{v=1}^{N} \sum_{k=1}^{N} \chi_{N_{A}} \chi_{N_{B}} u_{A}(k, n) \hat{x}(v) u_{B^{-1}}(k, v) \\
& =\sum_{k=1}^{N} \chi_{N_{A}} \sum_{v=1}^{N} \chi_{N_{B}} \hat{x}(v) u_{A B^{-1}}(n, v),
\end{aligned}
$$

Hence, according to the definition of the Frobenius matrix norm [1] and the definition of DLCT, we have

$$
\begin{aligned}
\left\|P_{N_{A}} P_{N_{B}}\right\|_{F} & =\left(\sum_{k=1}^{N} \chi_{N_{A}} \sum_{v=1}^{N} \chi_{N_{B}}\left|u_{A B^{-1}}(n, v)\right|^{2}\right)^{1 / 2} \\
& =\sqrt{\frac{N_{A} \cdot N_{B}}{N\left|a_{1} b_{2}-a_{2} b_{1}\right|}},
\end{aligned}
$$

In the similar manner with the continuous case, we can obtain $\frac{\left\|P_{N_{A}} P_{N_{B}} \tilde{x}(n)\right\|_{l^{2}(R)}}{\|\tilde{x}(n)\|_{l^{2}(R)}} \geq 1-\left(\varepsilon_{A}+\varepsilon_{B}\right)$. Since $\left\|P_{N_{A}} P_{N_{B}}\right\|_{F} \geq\left\|P_{N_{A}} P_{N_{B}}\right\|_{l^{2}(R)}=\sup _{x(n) \in l^{2}(R)} \frac{\left\|P_{N_{A}} P_{N_{B}} \tilde{x}(n)\right\|_{l^{2}(R)}}{\|\tilde{x}(n)\|_{l^{2}(R)}}$, we have 
$\sqrt{\frac{N_{A} \cdot N_{B}}{N\left|a_{1} b_{2}-a_{2} b_{1}\right|}}=\left\|P_{N_{A}} P_{N_{B}}\right\|_{F} \geq \frac{\left\|P_{N_{A}} P_{N_{B}} \tilde{x}(n)\right\|_{l^{2}(R)}}{\|\tilde{x}(n)\|_{l^{2}(R)}} \geq 1-\left(\varepsilon_{A}+\varepsilon_{B}\right)$, thus, we get $N_{A} \cdot N_{B} \geq N \cdot\left(1-\varepsilon_{A}-\varepsilon_{B}\right)^{2}\left|a_{1} b_{2}-a_{2} b_{1}\right|$. Therefore, we can obtain the following theorem 2.

Theorem 2: Let $\hat{x}_{A}(k)\left(\hat{x}_{B}(k)\right)$ be the DLCT of the time sequence $\tilde{x}(n) \in l^{2}(R)(n=1, \cdots, N)$ for transform parameter $A(B)$, with $\hat{x}_{A}(k)\left(\hat{x}_{B}(k)\right) \varepsilon_{A}\left(\varepsilon_{B}\right)$-concentrated on index set $N\left(\varepsilon_{A} \varepsilon_{B} \neq 0\right)$. Let $N_{A}\left(N_{B}\right)$ be the numbers of nonzero entries in $\hat{x}_{A}(k)\left(\hat{x}_{B}(k)\right.$ respectively). Then

$$
\begin{cases}N_{A} \cdot N_{B} \geq N \cdot\left(1-\varepsilon_{A}-\varepsilon_{B}\right)^{2}\left|a_{1} b_{2}-a_{2} b_{1}\right|, & a_{1} / b_{1} \neq a_{2} / b_{2} \\ N_{A} \cdot N_{B} \geq 1, & a_{1} / b_{1}=a_{2} / b_{2} .\end{cases}
$$

\subsection{Extensions}

Set $\varepsilon_{A}=\varepsilon_{B}=0$ in theorem 2 , we can obtain the following theorem 3 directly.

Theorem 3: Let $\hat{x}_{A}(k)\left(\hat{x}_{B}(k)\right)$ be the DLCT of the time sequence $\tilde{x}(n) \in l^{2}(R)(n=1, \cdots, N)$ with length $N . N_{A}\left(N_{B}\right)$ counts the numbers of nonzero entries in $\hat{x}_{A}(k)\left(\hat{x}_{B}(k)\right.$ respectively). Then

$$
\begin{cases}N_{A} \cdot N_{B} \geq N \cdot\left|a_{1} b_{2}-a_{2} b_{1}\right|, & a_{1} / b_{1} \neq a_{2} / b_{2} \\ N_{A} \cdot N_{B} \geq 1, & a_{1} / b_{1}=a_{2} / b_{2} .\end{cases}
$$

Clearly, theorem 3 is a special case of theorem 2. Also, this theorem can be derived via theorem 1 in [25]. Differently, we obtain this result in a different way. Here we note that since $\|\tilde{x}(n)\|_{l^{2}(R)}=1$, there is at least one non-zero element in every LCT domain for $a_{1} / b_{1}=a_{2} / b_{2}$. Therefore, $N_{A} \cdot N_{B} \geq 1$ for $a_{1} / b_{1}=a_{2} / b_{2}$. Through setting special value for $B=(1,0,0,1)$ in theorem 3 , we have

Corollary 1: $\left\{\begin{array}{ll}N_{A} \cdot N_{0} \geq N \cdot\left|b_{1}\right| & b_{1} \neq 0 \\ N_{A} \cdot N_{0} \geq 1 & b_{1}=0\end{array}\right.$ with $N_{0}=N_{(1,0,0,1)}$.

We can obtain the following more general uncertainty relation associated with DLCT.

Theorem 4: Let $\hat{x}_{A_{l}}(k)(l=1,2, \cdots, L)$ be the DLCT of the time sequence $\tilde{x}(n) \in l^{2}(R) \quad(n=1, \cdots, N$ and $N>L)$ with length $N$ and $\|\tilde{x}(n)\|_{l^{2}(R)}=1 . N_{\alpha_{l}}$ counts the number of nonzero elements in $\hat{x}_{A_{l}}(k)$. Then

$$
\frac{N_{A_{1}}+N_{A_{2}}+\cdots+N_{A_{L}}}{L} \geq \sqrt{N \cdot \xi} \text { with } \xi=\inf _{\substack{1 \leq l_{1}, l_{2} \leq L \\ l_{1} \neq l_{2}}}\left\{\left|a_{l_{1}} b_{l_{2}}-a_{l_{2}} b_{l_{1}}\right|\right\}
$$

Proof: From the assumption and the definition of DLCT [6], we know

$$
\tilde{x}(n)=\sum_{k_{1}=1}^{N} u_{\left(A_{1}\right)^{-1}}\left(n, k_{1}\right) \hat{x}_{A_{1}}\left(k_{1}\right)=\sum_{k_{2}=1}^{N} u_{\left(A_{2}\right)^{-1}}\left(n, k_{2}\right) \hat{x}_{A_{2}}\left(k_{2}\right)=\cdots=\sum_{k_{L}=1}^{N} u_{\left(A_{L}\right)^{-1}}\left(n, k_{L}\right) \hat{x}_{A_{L}}\left(k_{L}\right) \text { for } n=1,2, \cdots, N .
$$

where $u_{\left(A_{l}\right)^{-1}}\left(n, k_{l}\right)=\sqrt{-1 / i b_{l} N} \cdot \mathrm{e}^{\frac{-i a_{l} k_{l}^{2} \cot \alpha_{l}}{2 b_{l}}} \mathrm{e}^{\frac{i k_{l} n}{N b_{l}}} \mathrm{e}^{\frac{-i n^{2} d_{l}}{2 b_{l} N^{2}}}(l=1,2, \cdots, L)$. Therefore, let $\tilde{X}=[\tilde{x}(1), \tilde{x}(2), \cdots, \tilde{x}(N)]^{\mathrm{T}}$, have [25]

$$
\tilde{X}^{\mathrm{T}} \tilde{X}=\left[\hat{x}_{A_{1}}(1), \hat{x}_{A_{1}}(2), \cdots, \hat{x}_{A_{1}}(N)\right]\left[\begin{array}{c}
u_{\left(A_{l_{1}}\right)^{-1}}^{\mathrm{T}}(1,:) \\
u_{\left(A_{t_{1}}\right)^{-1}}^{\mathrm{T}}(2,:) \\
\vdots \\
u_{\left(A_{t_{1}}\right)^{-1}}^{\mathrm{T}}(N,:)
\end{array}\right]\left[u_{\left(A_{l_{2}}\right)^{-1}}(1,:), u_{\left(A_{l_{2}}\right)^{-1}}(2,:), \cdots, u_{\left(A_{t_{2}}\right)^{-1}}(N,:)\right]\left[\begin{array}{c}
\hat{x}_{\left(A_{l_{2}}\right)^{-1}}(1) \\
\hat{x}_{\left(A_{2}\right)^{-1}}(2) \\
\vdots \\
\hat{x}_{\left(A_{l_{2}}\right)^{-1}}(N)
\end{array}\right]
$$


where $u_{\left(A_{l_{1}}\right)^{-1}}^{\mathrm{T}}(n,:)=\left[u_{\left(A_{A_{1}}\right)^{-1}}(n, 1), u_{\left(A_{A_{1}}\right)^{-1}}(n, 2), \cdots, u_{\left(A_{l_{1}}\right)^{-1}}(n, N)\right]$ and $u_{\left(A_{l_{2}}\right)^{-1}}(n,:)=\left[u_{\left(A_{l_{2}}\right)^{-1}}(n, 1), u_{\left(A_{l_{2}}\right)^{-1}}(n, 2), \cdots, u_{\left(A_{l_{2}}\right)^{-1}}(n, N)\right]^{\mathrm{T}} \quad$ with $\quad n=1,2, \cdots, N \quad$ and $l_{1}, l_{2}=1,2, \cdots, L \quad$ with $l_{1} \neq l_{2}$.

Hence, we obtain

$$
\begin{aligned}
\tilde{X}^{\mathrm{T}} \tilde{X} & =\left[\hat{x}_{A_{l_{1}}}(1), \hat{x}_{A_{l_{1}}}(2), \cdots, \hat{x}_{A_{l_{1}}}(N)\right] \\
& =\sum_{n=1}^{N} \sum_{k=1}^{N} \hat{x}_{A_{l_{1}}}(n)\left\langle u_{\left(A_{l_{1}}\right)^{-1}}(n,:), u_{\left(A_{l_{2}}\right)^{-1}}(k,:)\right) \hat{x}_{A_{l_{2}}}(k),
\end{aligned}
$$

Set $M_{\left(l_{1}, l_{2}\right)}=\sup _{n, k}\left(\mid\left\langle u_{\left(A_{l_{1}}\right)^{-1}}(n,:), u_{\left(A_{l_{2}}\right)^{-1}}(k,:)\right\rangle\right)$, then

$$
\begin{aligned}
\tilde{X}^{\mathrm{T}} \tilde{X} & \leq \sum_{n=1}^{N} \sum_{k=1}^{N}\left|\hat{x}_{A_{l_{1}}}(n)\left\langle u_{\left(A_{l_{1}}\right)^{-1}}(n,:), u_{\left(A_{l_{2}}\right)^{-1}}(k,:)\right\rangle \hat{x}_{A_{l_{2}}}(k)\right| \\
& \leq M_{\left(l_{1}, l_{2}\right)} \sum_{s_{1}=1}^{N_{A_{1}}{ }_{A_{A_{2}}}} \sum_{s_{2}=1}\left|\hat{x}_{A_{l_{1}}}\left(s_{1}\right)\right| \cdot\left|\hat{x}_{A_{l_{2}}}\left(s_{2}\right)\right|
\end{aligned}
$$

Using the triangle inequality, we have

$$
\left|\hat{x}_{A_{l_{1}}}\left(s_{1}\right)\right| \cdot\left|\hat{x}_{A_{l_{2}}}\left(s_{2}\right)\right| \leq \frac{\left|\hat{x}_{A_{1}}\left(s_{1}\right)\right|^{2}+\left|\hat{x}_{A_{l_{2}}}\left(s_{2}\right)\right|^{2}}{2}
$$

hence

From $\|\tilde{x}(n)\|_{2}=1$ and Parseval's principle [6], we obtain:

$$
\sum_{s_{1}=1}^{N_{A_{1}}} \frac{\left|\hat{x}_{A_{1}}\left(s_{1}\right)\right|^{2}}{2}=\sum_{s_{2}=1}^{N_{\alpha_{l_{2}}}} \frac{\left|\hat{x}_{A_{l_{2}}}\left(s_{2}\right)\right|^{2}}{2}=\frac{1}{2}
$$

Hence

$$
\tilde{X}^{\mathrm{T}} \tilde{X} \leq M_{\left(l_{1}, l_{2}\right)} \cdot\left(\sum_{s_{2}=1}^{N_{A_{l_{2}}}} \frac{1}{2}+\sum_{s_{1}=1}^{N_{A_{1}}} \frac{1}{2}\right)=M_{\left(l_{1}, l_{2}\right)} \cdot \frac{N_{A_{l_{1}}}+N_{A_{l_{2}}}}{2} .
$$

Therefore, we obtain

$$
\begin{aligned}
& \tilde{X}^{\mathrm{T}} \tilde{X} \leq M_{(1,2)} \cdot \frac{N_{1}+N_{2}}{2}, \\
& \tilde{X}^{\mathrm{T}} \tilde{X} \leq M_{(1,3)} \cdot \frac{N_{1}+N_{3}}{2}, \\
& \vdots \\
& \tilde{X}^{\mathrm{T}} \tilde{X} \leq M_{(L-1, L)} \cdot \frac{N_{L-1}+N_{L}}{2} .
\end{aligned}
$$

Adding all the above inequalities, we have $\Gamma_{L}^{2} \cdot \tilde{X}^{\mathrm{T}} \tilde{X} \leq \sup _{\substack{1 \leq l_{1}, l_{2} \leq L \\ l_{1} \neq l_{2}}}\left\{M_{\left(l_{1}, l_{2}\right)}\right\} \frac{(L-1) \cdot\left(N_{1}+N_{2}+\cdots+N_{L}\right)}{2}$ with $\Gamma_{L}^{2}=\frac{L \cdot(L-1)}{2 \times 1}$. Similarly, from $\|\tilde{x}(n)\|_{2}=1$ and Parseval's principle [6], we obtain $\tilde{X}^{\mathrm{T}} \tilde{X}=1$, hence 


$$
\frac{(L-1) \cdot\left(N_{1}+N_{2}+\cdots+N_{L}\right)}{2} \geq \frac{\Gamma_{L}^{2} \cdot}{\sup _{\substack{1 \leq l_{1}, l_{2} \leq L \\ l_{1} \neq l_{2}}}\left\{M_{\left(l_{1}, l_{2}\right)}\right\}} .
$$

From the definition and property of DLCT [6] we have

$$
\begin{aligned}
\sup _{\substack{1 \leq l_{1}, l_{2} \leq L \\
l_{1} \neq l_{2}}}\left\{M_{\left(l_{1}, l_{2}\right)}\right\} & =\sup _{\substack{1 \leq s_{1}, s_{2} \leq N \\
1 \leq l_{1}, l_{2} \leq L, l_{1} \neq l_{2}}}\left(\left|K_{\left(A_{l_{1}}\right)^{-1} A_{l_{2}}}\left(s_{1}, s_{2}\right)\right|\right) \\
& \left.=\sup _{\substack{1 \leq s_{1}, s_{2} \leq N \\
1 \leq l_{1}, l_{2} \leq L, l_{1} \neq l_{2}}}|| \frac{1}{\sqrt{N \cdot\left|a_{l_{1}} b_{l_{2}}-a_{l_{2}} b_{l_{1}}\right|} \mid} \mid\right)=\frac{1}{\sqrt{N \cdot \xi}} .
\end{aligned}
$$

with $\xi=\inf _{\substack{1 \leq l_{1}, l_{2} \leq L \\ l_{1} \neq l_{2}}}\left\{\left|a_{l_{1}} b_{l_{2}}-a_{l_{2}} b_{l_{1}}\right|\right\}$.

Hence, we finally obtain $\frac{N_{1}+N_{2}+\cdots+N_{L}}{L} \geq \sqrt{N \cdot \xi}$ with $\xi=\inf _{\substack{1 \leq l_{1}, l_{2} \leq L \\ l_{1} \neq l_{2}}}\left\{\left|a_{l_{1}} b_{l_{2}}-a_{l_{2}} b_{l_{1}}\right|\right\}$. This theorem is the extension of theorem 3 and discloses the uncertainty relation between multiple signals.

\section{Conclusion}

In practice, for the discrete data, not only the supports are limited, but also they are sequences of data points whose number of non-zero elements is countable accurately. This paper discussed the generalized uncertainty relations on LCT in terms of data concentration. We show that the uncertainty bounds are related to the LCT parameters and the support lengths. These uncertainty relations will enrich the ensemble of uncertainty principles and yield the potential illumination for physics.

\section{Acknowledgements}

This work was fully supported by the NSFCs (61002052 and 61471412) and partly supported by the NSFC (61250006).

\section{Supported}

This work was fully supported by the NSFC (61002052) and partly supported by the NSFC (61250006 and 60975016).

\section{References}

[1] Zhang, X.D. (2002) Modern Signal Processing. 2nd Edition, Tsinghua University Press, Beijing, 362.

[2] Selig, K.K. (2002) Uncertainty Principles Revisited. Electronic Transactions on Numerical Analysis, 14, 165-177.

[3] Dembo, A., Cover, T.M. and Thomas, J.A. (2001) Information Theoretic Inequalities. IEEE Transactions on Information Theory, 37, 1501-1508.

[4] Loughlin, P.J. and Cohen, L. (2004) The Uncertainty Principle: Global, Local, or Both? IEEE Transactions on Signal Processing, 52, 1218-1227.

[5] Folland, G.B. and Sitaram, A. (1997) The Uncertainty Principle: A Mathematical Survey. The Journal of Fourier Analysis and Applications, 3, 207-238. http://dx.doi.org/10.1007/BF02649110

[6] Tao, R., Deng, B. and Wang, Y. (2009) Theory and Application of the Fractional Fourier Transform. Tsinghua University Press, Beijing.

[7] Maassen, H. (1988) A Discrete Entropic Uncertainty Relation. Quantum Probability and Applications, Springer-Verlag, New York, 263-266.

[8] Stern, A. (2007) Sampling of Compact Signals in Offset Linear Canonical Transform Domains. Signal, Image and Video Processing, 1, 359-367.

[9] Shinde, S. and Vikram, M.G. (2001) An Uncertainty Principle for Real Signals in the Fractional Fourier Transform 
Domain. IEEE Transactions on Signal Processing, 49, 2545-2548.

[10] Mustard, D. (1991) Uncertainty Principle Invariant under Fractional Fourier Transform. The Journal of the Australian Mathematical Society. Series B. Applied Mathematics, 33, 180-191. http://dx.doi.org/10.1017/S0334270000006986

[11] Bialynicki-Birula, I. (1985) Entropic Uncertainty Relations in Quantum Mechanics. In: Accardi, L. and von Waldenfels, W., Eds., Quantum Probability and Applications II, Lecture Notes in Mathematics, Volume 1136, Springer, Berlin 90.

[12] Aytür, O. and Ozaktas, H.M. (1995) Non-Orthogonal Domains in Phase Space of Quantum Optics and Their Relation to Fractional Fourier Transforms. Optics Communications, 120, 166-170. http://dx.doi.org/10.1016/0030-4018(95)00452-E

[13] Stern, A. (2008) Uncertainty Principles in Linear Canonical Transform Domains and Some of Their Implications in Optics. Journal of the Optical Society of America A, 25, 647-652. http://dx.doi.org/10.1364/JOSAA.25.000647

[14] Sharma, K.K. and Joshi, S.D. (2008) Uncertainty Principle for Real Signals in the Linear Canonical Transform Domains. IEEE Transactions on Signal Processing, 56, 2677-2683. http://dx.doi.org/10.1109/TSP.2008.917384

[15] Zhao, J., Tao, R., Li, Y.L. and Wang, Y. (2009) Uncertainty Principles for Linear Canonical Transform. IEEE Transactions on Signal Processing, 57, 2856-2858. http://dx.doi.org/10.1109/TSP.2009.2020039

[16] Xu, G.L., Wang, X.T. and Xu, X.G. (2009) Three Cases of Uncertainty Principle for Real Signals in Linear Canonical Transform Domain. IET Signal Processing, 3, 85-92. http://dx.doi.org/10.1049/iet-spr:20080019

[17] Xu, G.L., Wang, X.T. and Xu, X.G. (2009) New Inequalities and Uncertainty Relations on Linear Canonical Transform Revisit. EURASIP Journal on Advances in Signal Processing, 2009, Article ID: 563265. http://dx.doi.org/10.1155/2009/563265

[18] Xu, G.L., Wang, X.T. and Xu, X.G. (2009) Generalized Entropic Uncertainty Principle on Fractional Fourier Transform. Signal Processing, 89, 2692-2697. http://dx.doi.org/10.1016/j.sigpro.2009.05.014

[19] Xu, G.L., Wang, X.T. and Xu, X.G. (2009) Uncertainty Inequalities for Linear Canonical Transform. IET Signal Processing, 3, 392-402. http://dx.doi.org/10.1049/iet-spr.2008.0102

[20] Xu, G.L., Wang, X.T. and Xu, X.G. (2009) The Logarithmic, Heisenberg's and Short-Time Uncertainty Principles Associated with Fractional Fourier Transform. Signal Processing, 89, 339-343. http://dx.doi.org/10.1016/j.sigpro.2008.09.002

[21] Xu, G.L., Wang, X.T. and Xu, X.G. (2010) On Uncertainty Principle for the Linear Canonical Transform of Complex Signals. IEEE Transactions on Signal Processing, 58, 4916-4918. http://dx.doi.org/10.1109/TSP.2010.2050201

[22] Somaraju, R. and Hanlen, L.W. (2006) Uncertainty Principles for Signal Concentrations. Proceedings of the 7th Australian Communications Theory Workshop, Perth, 1-3 February 2006, 38-42. http://dx.doi.org/10.1109/AUSCTW.2006.1625252

[23] Donoho, D.L. and Huo, X. (2001) Uncertainty Principles and Ideal Atomic Decomposition. IEEE Transactions on Information Theory, 47, 2845-2862. http://dx.doi.org/10.1109/18.959265

[24] Donoho, D.L. and Stark, P.B. (1989) Uncertainty Principles and Signal Recovery. SIAM Journal on Applied Mathematics, 49, 906-930. http://dx.doi.org/10.1137/0149053

[25] Elad, M. and Bruckstein, A.M. (2002) A Generalized Uncertainty Principle and Sparse Representation in Pairs of Bases. IEEE Transactions on Information Theory, 48, 2558-2567. http://dx.doi.org/10.1109/TIT.2002.801410

[26] Xu, G.L., Wang, X.T. and Xu, X.G. (2010) Novel Uncertainty Relations in Fractional Fourier Transform Domain for Real Signals. Chinese Physics B, 19, Article ID: 014203. http://dx.doi.org/10.1088/1674-1056/19/1/014203

[27] Pei, S.C., Yeh, M.H. and Luo, T.L. (1999) Fractional Fourier Series Expansion for Finite Signals and Dual Extension to Discrete-Time Fractional Fourier Transform. IEEE Transactions on Circuits and System II: Analog and Digital Signal Processing, 47, 2883-2888.

[28] Pei, S.C. and Ding, J.J. (2003) Eigenfunctions of the Offset Fourier, Fractional Fourier, and Linear Canonical Transforms. Journal of the Optical Society of America A, 20, 522-532. http://dx.doi.org/10.1364/JOSAA.20.000522

[29] Qi, L., Tao, R., Zhou, S. and Wang, Y. (2004) Detection and Parameter Estimation of Multicomponent LFM Signal Based on the Fractional Fourier Transform. Science in China Series F, 47, 184-198. http://dx.doi.org/10.1360/02yf0456 